\title{
Correlations in prompt neutrons and gamma-rays from Cf-252 spontaneous fission
}

\author{
M.J. Marcath ${ }^{\mathrm{a}}$, T.H. Shin, A. Di Fulvio, S.D. Clarke, and S.A. Pozzi
}

Nuclear Engineering and Radiological Sciences Department, University of Michigan, 2355 Bonisteel Blvd, Ann Arbor, MI 48109, USA

\begin{abstract}
New event-by-event fission models have prompt neutrons and gamma-rays that are correlated in time, energy, and multiplicity, however there is limited measurement data available to validate these models. Measurement of high-order fission neutron and gamma-ray coincidences is difficult and there has previously been little motivation to measure properties of both particle types simultaneously. High-order Cf252 spontaneous fission neutron and gamma-ray coincidences were measured with a cylindrical array of 22 liquid organic and $8 \mathrm{NaI}(\mathrm{Tl})$ scintillation detectors, $50 \mathrm{~cm}$ from a central axis. Waveforms were acquired and saved for post-processing using four time-synchronized CAEN V1720 digitizers. Liquid organic scintillator waveforms were analyzed with off-line pulse shape discrimination techniques to categorize neutron and gamma-ray detections. Detected multiplicity was compared with MCNPX-PoliMi simulation results, where built-in fission models and event-by-event fission models, CGMF and FREYA, have been implemented. Additionally, measured neutron energy by time-of-flight and gamma-ray energy correlated by detected multiplicity were compared to simulated results.
\end{abstract}

\section{Background}

Improved understanding of prompt fission emissions could lead to new safeguards and verification measurement techniques. The physics community is also motivated to improve the theory of prompt neutron and gammaray emission, particularly to characterize correlation between those particles. Recently developed event-byevent physics based models CGMF and FREYA make predictions of correlations [1,2], which can now be tested. Many studies of prompt emissions have already been done, exclusive to one particle type; however this work seeks to characterize the correlation between Cf252 spontaneous fission neutrons and gamma-rays and to compare simulated results, with various fission models invoked, to measured results.

\section{Methods}

Shown in Fig. 1, an array of 14-7.62 $\times 7.62 \mathrm{~cm} \mathrm{EJ-}$ 309 's, 8-7.62 × 5.08 cm EJ-309's, and 8-7.62Ø×7.62 cm sodium iodide ( $\mathrm{NaI}(\mathrm{Tl})$ ) scintillators coupled to photomultiplier tubes was used to measure neutrons and gammarays from $\mathrm{Cf}-252$ spontaneous fission. The detectors were aligned so that there were two horizontal planes of detectors separated by $20 \mathrm{~cm}$ from detector center axes. Detectors on each plane are in $20^{\circ}$ increments. A sealed 38,000 fission/s Cf-252 spontaneous fission source was placed on the center axis between the two detector planes. The array flightpath was $51 \mathrm{~cm}$ for all detectors.

A dedicated background measurement was performed to subtract chance events from the Cf-252 spontaneous fission signals.

a e-mail: mmarcath@umich.edu
Pulses from the detectors were digitized using four CAEN V1720 waveform digitizers with $250 \mathrm{MHz}$ sampling and 12 bit amplitude resolution over a $2 \mathrm{~V}$ range. The detectors were gain matched to $478 \mathrm{keVee}$ at $0.3 \mathrm{~V}$ with a threshold of $70 \mathrm{keVee}$. All digitized waveforms were recorded for post-processing.

In post-processing, waveforms were removed where pulse shape discrimination (PSD) or timing calculations would be unreliable. Double pulses above a fraction of the triggering pulse height are removed. The double pulse fractional height threshold is $10 \%$ for EJ-309's and $20 \%$ for the $\mathrm{NaI}(\mathrm{Tl})$ 's. Pulse timing is determined through constant fraction discrimination (CFD).

EJ-309 organic liquid is pulse shape discrimination capable. The pulse shape of neutron and photon interactions differ significantly enough to discriminate particle detection type [3]. Pulse shape discrimination by tail and total waveform integrals was used to discriminate between neutron and gamma-ray detection in the liquid organic scintillators [4]. A quadratic line is used to discriminate particle type, shown in Fig. 2. At low pulse heights, pulse shape discrimination becomes challenging and is subject to misclassification errors.

After post-processing of waveforms including pulse shape discrimination, coincident detections using a $200 \mathrm{~ns}$ window were collected. The coincident detections were analyzed to produce pulse height, cross-correlation, multiplicity, and time-of-flight neutron energy distributions. The distributions can be compared to simulated results with various fission models.

MCNPX-PoliMi was used to simulate the measurement and MPPost was used to analyze data to replicate detector response [5,6]. Simulated MCNPX-PoliMi results using the built-in Cf-252 spontaneous fission model, the CGMF model, and the FREYA model are compared to

(C) The Authors, published by EDP Sciences. This is an Open Access article distributed under the terms of the Creative Commons Attribution License 4.0 (http://creativecommons.org/licenses/by/4.0/). 


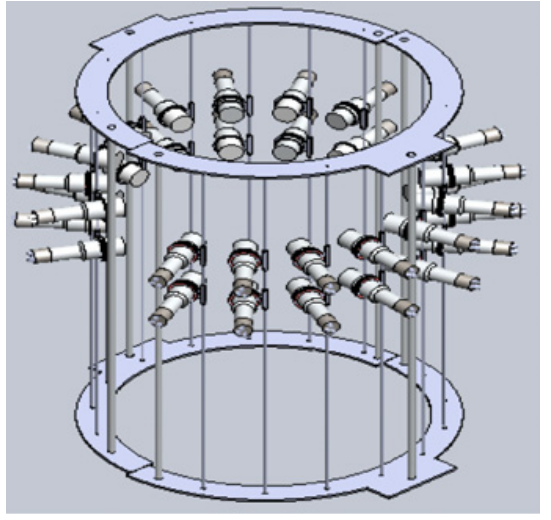

Figure 1. A model of the support structure and 30 detectors, $14-7.62 \times 7.62 \mathrm{~cm}$ EJ-309's, 8-7.62 × 5.08 cm EJ-309's, and $8-7.62 \varnothing \times 7.62 \mathrm{~cm}$ sodium iodide $(\mathrm{NaI}(\mathrm{Tl}))$ scintillators. Two detectors shown here were removed.

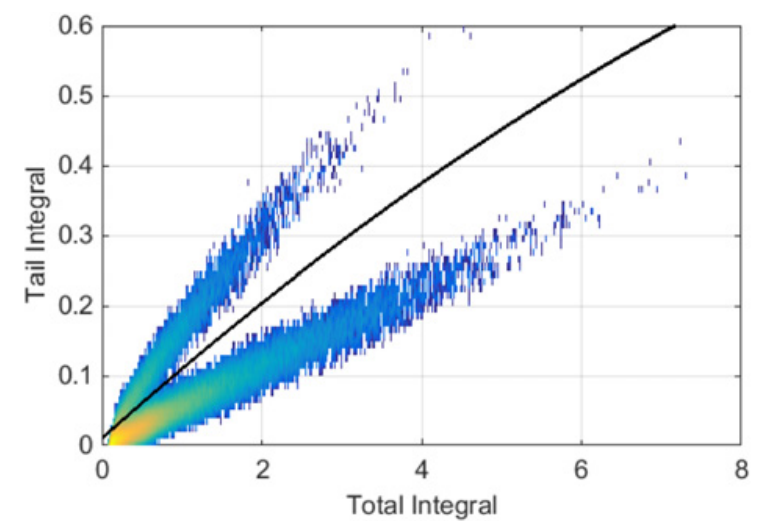

Figure 2. EJ-309 digitized waveform pulse tail integral to total pulse integral heat map with neutron-gamma-ray discrimination line. Approximately 200,000 waveforms, after cleaning, are represented in the figure.

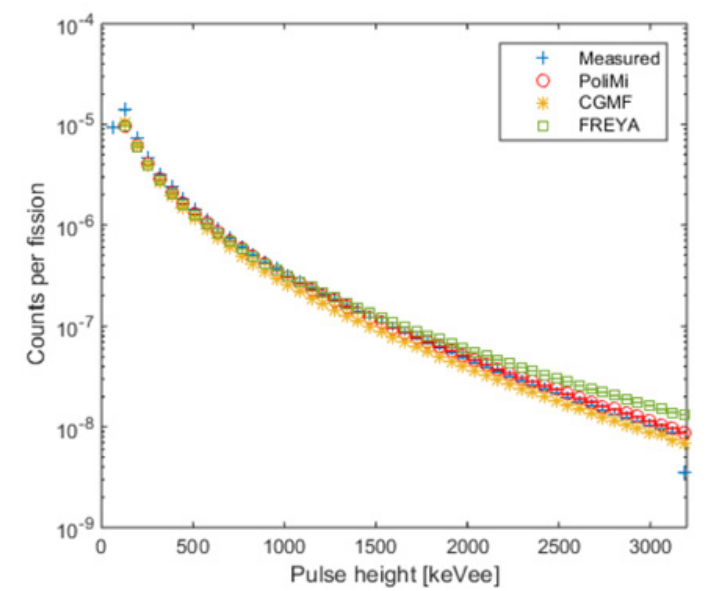

Figure 3. $7.62 \varnothing \times 7.62 \mathrm{~cm}$ EJ-309 neutron pulse height histogram.

measured results. The built-in MCNPX-PoliMi source does not exhibit neutron and gamma-ray correlation, whereas both CGMF and FREYA do. The event-byevent CGMF and FREYA algorithms handle the transition from primarily neutron emission to gamma-ray emission differently resulting in differences in observed particle correlations.

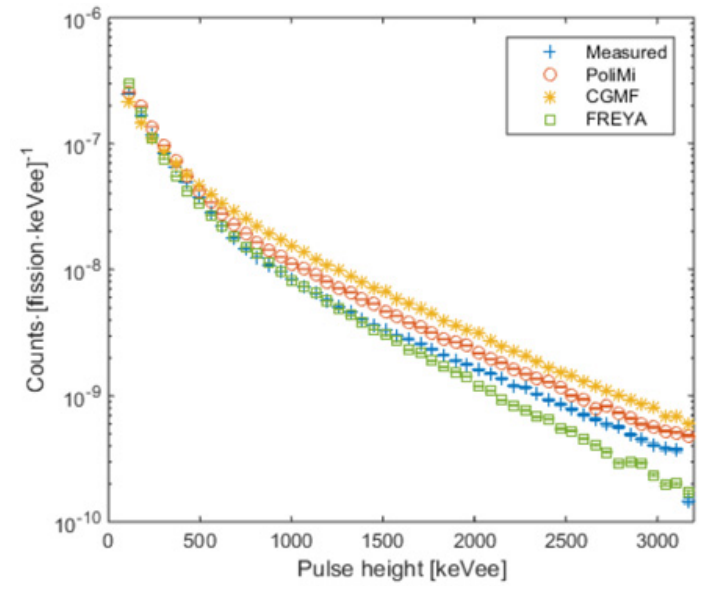

Figure 4. $7.62 \varnothing \times 7.62 \mathrm{~cm}$ EJ-309 photon pulse height histogram with a neutron coincidence required 5 to $75 \mathrm{~ns}$ after the photon detection.

MPPost was used to emulate detector response. MCNPX-PoliMi collision histories are read and evaluated by MPPost to determine the outcome of the interaction and relevant information is recorded. The user provides detection thresholds and resolutions to be applied by MPPost.

\section{Results}

Measurement and simulation analysis were performed with analogous methods so similar quantities could be directly compared. The MCNPX-PoliMi code was used to read fission histories from CGMF, FREYA, and its builtin Cf-252 models. After measurement post-processing and simulation collision file processing with MPPost, key measurement quantities are recorded and compared. Pulse height and cross-correlation histogram agreement help to demonstrate basic detector response and geometry accuracy, but higher order quantities are compared to investigate fission model differences.

\subsection{MCNPX-PoliMi model and detector response validation}

The singles pulse height histograms from the measurement and simulations are shown in Figs. 3 and 4. The histograms are the average of the $7.62 \varnothing \times 7.62 \mathrm{~cm}$ EJ-309 detector set. The histograms show the background subtracted pulse height spectra, after PSD in the case of EJ-309s. Pulse height is a convolution of the detector response and the incident spectra; the light output and the cross-section in neutron scattering on protons is non-linear, resulting in a pulse height distribution that doesn't resemble the incident Maxwellian spectrum. Non-fission emissions from fission products built up in the Cf-252 sample are included in these spectra; these emissions are primarily photons.

In both pulse height histograms, the discrepancy at the lowest pulse heights is due to a mis-match in the detection threshold. The neutron pulse height spectra show good agreement between all models, but above approximately 1,500 keVee the FREYA model over-predicts the measured result. Above $1500 \mathrm{keVee}$, the models diverge slightly from the experimental data. 


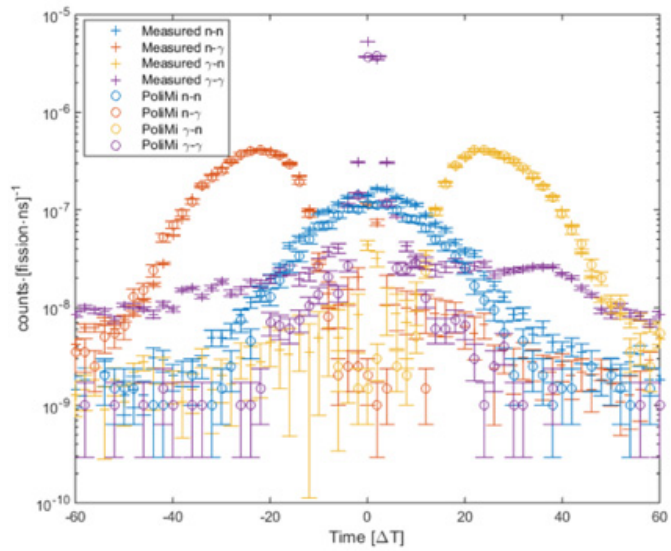

Figure 5. Cross-correlation distribution between two $7.62 \varnothing \times$ $7.62 \mathrm{~cm}$ EJ-309 detectors with 134 degrees between the detectors.

Conditioning photon pulse height histogramming on a coincident neutron detection improves measurementsimulation pulse height histogram agreement, shown in Fig. 4. The coincidence condition does not impact the shape of the simulation histograms significantly, whereas the measurement histograms are improved. Change in the measurement histogram shape indicates that non-fission emissions are contributing to the histogram. FREYA under-predicts while the built-in PoliMi model and CGMF over-predict the measured result over most of the range.

Comparison of time cross-correlations show good agreement between measured and MCNPX-PoliMi simulated results. Figure 5 shows a cross-correlation time distribution for neutron-neutron, neutron-photon, photon-neutron, and photon-photon coincidences for both measured and simulated results. The photon-photon distributions disagree outside of approximately $+-5 \mathrm{~ns}$ due to non-fission, un-modeled photon emissions from the source material which are not entirely removed through simple background subtraction.

\subsection{Correlated results}

\subsubsection{Coincidences}

Coincidence tallies were recorded for a $80 \mathrm{~ns}$ window for each simulation model and the measured data. Events were tallied when two or more channels trigger within a $80 \mathrm{~ns}$ window. The coincidences were binned by the number of neutrons detected within the coincidence window in Figs. 6 and 7. Neutron coincidence tally results are shown in Fig. 6; simulation results for all models over-predict the number of coincidences for all neutron coincidences except zero. Despite background subtraction, the number of coincidences is underpredicted at zero because background photon coincidences contribute disproportionately. The likely case is a real Cf-252 fission photon is detected in coincidence with a background photon. CGMF agrees best over the range of coincidences, but no model agrees well at high neutron coincidences. Agreement becomes progressively worse as order of coincidence increases.

Average photon coincidences as a function of neutron coincidences the measured number of coincidences is shown in Fig. 7. The fission models with neutron and gamma-ray correlation underestimate the measured result,

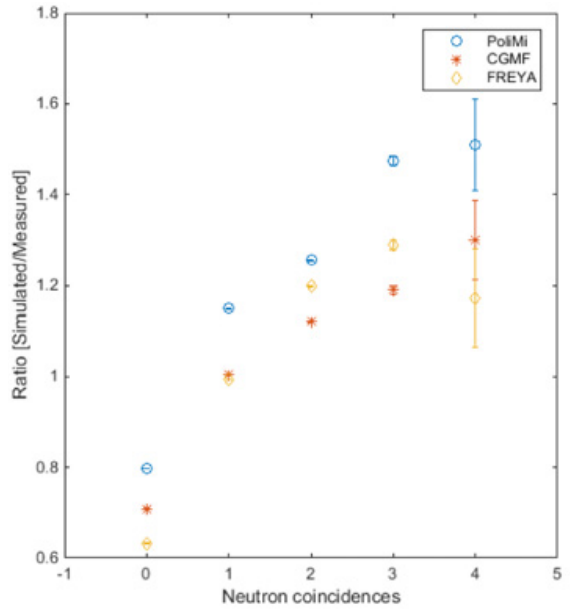

Figure 6. Ratio of neutron coincidence tallies, simulation to measurement.

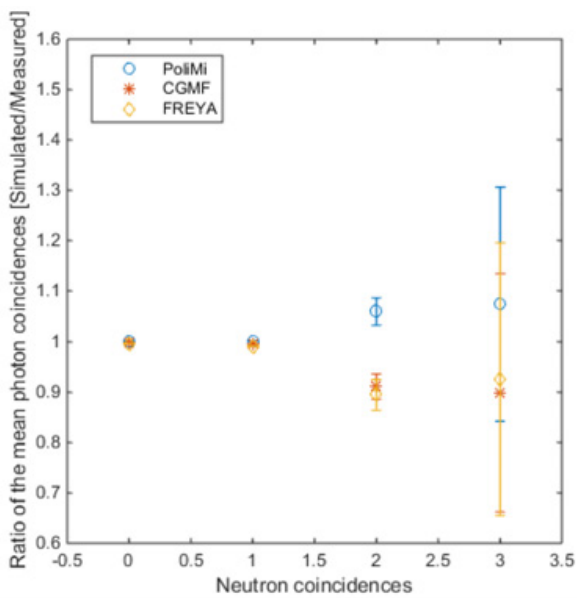

Figure 7. Average photon coincidences as a function of neutron coincidences.

while the built-in PoliMi model with no correlation overpredicts measurement.

\subsubsection{Neutron time-of-flight}

Neutron energy spectra were estimated through time-offlight techniques. The time difference of a photon detection in an EJ-309 and neutron detection in a different EJ-309 was converted to neutron energy. Timing resolution is the largest contributor to uncertainty in the estimate of neutron energy, especially for high energy neutrons. Delayed and scattered photons also contribute to uncertainty in the neutron energy. The energy spectra histograms are corrected for detector efficiency to estimate the incident neutron spectra.

Tables 1 and 2 show mean neutron energy as a function of coincident detections for measured and simulated data. A slight increase in neutron energy is observed with the number of coincident detections in table I and II. More data is required to resolve any trend in the neutron energy; the upward trend is generally small relative to the statistical uncertainties. 
Table 1. Average detected neutron energy by time-of-flight over the sensitive range of the detectors, $1.1-8.1 \mathrm{MeV}$, as a function of neutron coincidences. Omitted entries had insufficient data.

\begin{tabular}{|c|c|c|c|c|}
\hline \multirow{2}{*}{$\begin{array}{l}\text { Number } \\
\text { of } \\
\text { detected } \\
\text { neutrons }\end{array}$} & \multicolumn{4}{|c|}{ Fission model } \\
\hline & PoliMi & CGMF & FREYA & Measured \\
\hline 1 & $2.492(2)$ & $2.359(1)$ & $2.563(2)$ & $2.668(2)$ \\
\hline 2 & $2.51(1)$ & $2.422(8)$ & $2.61(1)$ & $2.72(1)$ \\
\hline 3 & $2.49(6)$ & $2.49(6)$ & $2.63(8)$ & $2.7(1)$ \\
\hline 4 & $2.5(6)$ & $2.3(5)$ & $3.2(9)$ & - \\
\hline
\end{tabular}

Table 2. Average detected neutron energy by time-of-flight over the sensitive range of the detectors, $1.1-48.1 \mathrm{MeV}$, as a function of photon coincidences. Omitted entries had insufficient data.

\begin{tabular}{|c|c|c|c|c|}
\hline \multirow{2}{*}{$\begin{array}{c}\text { Number } \\
\text { of } \\
\text { detected } \\
\text { photons }\end{array}$} & PoliMi & CGMF & FREYA & Measured \\
\cline { 2 - 5 } & $2.492(2)$ & $2.359(1)$ & $2.563(2)$ & $2.668(2)$ \\
\hline 1 & $2.51(1)$ & $2.422(8)$ & $2.61(1)$ & $2.72(1)$ \\
\hline 2 & $2.49(6)$ & $2.49(6)$ & $2.63(8)$ & $2.7(1)$ \\
\hline 3 & $2.5(6)$ & $2.3(5)$ & $3.2(9)$ & - \\
\hline 4 & \multicolumn{4}{|c|}{ Fission model } \\
\hline
\end{tabular}

\section{Conclusions}

Detailed and complex event-by-event fission treatments are being developed to more accurately represent the fission process, including neutron and gamma-ray correlations. The EJ-309 liquid organic and $\mathrm{NaI}(\mathrm{Tl})$ array measured high-order Cf-252 spontaneous fission prompt neutron and gamma-ray correlated quantities. Neutron time-of-flight data was analyzed to find neutron energy correlated data distributions. With MCNPXPoliMi simulations, these fission model neutron and gamma-ray correlations were compared with measured Cf252 spontaneous fission data.

Cross-correlation and pulse height distributions for measured and simulated results agree well, except for gamma-ray pulse height distributions due to non-fission gamma-ray sources present in measurement that were not modeled. Trends in the number of particles detected in coincidence compare well with measured data, however all models over-predicted the average number of neutrons detected in coincidence with more than one gammaray. No significant trend larger than the uncertainty in neutron energy was observed with the number of particles detected in coincidence. Future work should seek to increase sensitivity to high order correlations while maintaining neutron time-of-flight ability to better discriminate between fission models.

\section{References}

[1] P. Talou, I. Stetcu, and T. Kawano, "Modeling the Emission of Prompt Fission Gamma-rays for Fundamental Physics Applications," Phys. Procedia, 69, 83-88 (2014)

[2] R. Vogt and J. Randrup, "Event-by-event study of photon observables in spontaneous and thermal fission," Phys. Rev. C 87, 044602 (2013)

[3] F.D. Brooks, R. W. Pringle, B. L. Funt, "Pulse Shape Discrimination in a Plastic Scintillator," Trans. Nucl. Sci. 7(2-3), 35-38 (1960)

[4] G. F. Knoll, Radiation Detection and Measurement, $3^{\text {rd }}$ ed., New York: John Wiley (2000), pp. 230-231

[5] S. A. Pozzi et al., "MCNP-PoliMi: A Monte Carlo Code for Correlation Measurements," Nucl. Instrum. Methods Phys. Res., Sect. A 513, 550 (2003)

[6] X-5 Monte Carlo Team, "MCNP-A general NParticle Transport Code, Version 5, Volume I: Overview and Theory," LA-UR-03-1987, Los Alamos National Laboratory (2003) 